\title{
Long Cycles in 3-Connected Graphs in Orientable Surfaces*
}

\author{
Laura Sheppardson \\ Xingxing $\mathrm{Yu}^{\dagger}$ \\ School of Mathematics \\ Georgia Institute of Technology \\ Atlanta, Georgia 30332
}

August 16, 2000

\begin{abstract}
In this paper we apply a cutting theorem of Thomassen to show that there is a function $f: \mathbf{N} \rightarrow \mathbf{N}$ such that if $G$ is a 3-connected graph which can be embedded in the orientable surface of genus $g$ with face-width at least $f(g)$, then $G$ contains a cycle of length at least $c\left(n^{\log _{3} 2}\right)$, where $c$ is a constant not dependent on $g$.
\end{abstract}

*MSC Primary 05C38 and 05C50 Secondary 57M15

${ }^{\dagger}$ Partially supported by NSF grant DMS-9970527 


\section{Introduction}

Whitney [11] proved in 1931 that every 4-connected triangulation of the plane contains a Hamilton cycle. In 1956, Tutte [9] showed that in fact all 4-connected planar graphs are Hamiltonian. Thomas and Yu [7] have extended this to show that 4-connected projective planar graphs are also Hamiltonian. Archdeacon, Hartsfield, and Little [1] showed that this result does not extend to graphs embedded in other surfaces. They proved that for each $n$, there is a triangulation of an orientable surface which is $n$-connected and in which every spanning tree contains a vertex of degree at least $n$. (Note that a Hamilton path is a spanning tree with maximum degree two.) However, in any 5-connected triangulation of surfaces with large face-width (see definition below), Yu [12] proved that there is a Hamiltonian path. (This is still open for non-triangulations.)

We also lose Hamiltonicity in planar graphs if we relax the connectivity condition. Moon and Moser [6] showed that there exist 3-connected planar triangulations on $n$ vertices whose longest cycles are of length $O\left(n^{\log _{3} 2}\right)$. They conjectured, however, that any 3 -connected planar graph on $n$ vertices must contain a cycle of length at least $O\left(n^{\log _{3} 2}\right)$. Chen and $\mathrm{Yu}[3]$ recently proved this conjecture, not only for planar graphs, but also for graphs embeddable in the projective plane, or the torus, or the Klein Bottle.

This result can be extended to graphs on other surfaces under certain conditions. The face-width (or representativity) of a graph $G$ embedded in a surface $\Sigma$ is defined to be the number $\min \{|\Gamma \cap V(G)|: \Gamma$ is a homotopically nontrivial closed curve in $\Sigma$, and $\Gamma \cap G \subset V(G)\}$. We will show that if a graph on $n$ vertices is embeddable in some fixed orientable surface with sufficiently large face-width, then it contains a cycle of length at least $c n^{\log _{3} 2}$, where $c=\frac{1}{2}\left(\frac{1}{4}\right)^{\log _{3} 2}$.

The techniques used here are similar to those in [3], combined with a cutting technique of Thomassen in [8]. In this paper, we first review the definitions to be used in graph cutting and the necessary lemmas. Our main theorem is stated 
following these definitions. We then define circuit graphs and annulus graphs, special classes of 2-connected planar graphs. We show the existence of "heavy" paths in vertex-weighted circuit or annulus graphs. Finally, the previous results are combined to prove the main theorem. The theorem addresses "heavy" cycles in vertex-weighted graphs, which easily extends to long cycles by applying a uniform weighting.

Graph Cutting If $H$ is a graph embedded in an orientable surface $\Sigma$, and $C$ is a cycle in $H$, we talk about cutting $H$ and $\Sigma$ along $C$. To cut along $C$, where $C=x_{1} x_{2} \ldots x_{k} x_{1}$ is an orientation preserving cycle, we add cycle $C^{\prime}=x_{1}^{\prime} x_{2}^{\prime} \ldots x_{k}^{\prime} x_{1}^{\prime}$ immediately to the left of $C$. Where edge $v x_{i}$ is incident with $C$ on its left, we remove $v x_{i}$ and replace it with edge $v x_{i}^{\prime}$. We then remove the open region of $\Sigma$ between $C$ and $C^{\prime}$, and attach a disc to each of $C$ and $C^{\prime}$. This yields a new graph $H^{\prime}$ embedded in a new surface $\Sigma^{\prime}$, or new graphs $H_{1}^{\prime}$ and $H_{2}^{\prime}$ embedded in surfaces $\Sigma_{1}^{\prime}$ and $\Sigma_{2}^{\prime}$, respectively.

Let $H$ be a graph embedded in some surface $\Sigma$, and let $C_{0}$ and $C_{1}$ be two disjoint cycles in $H$ which are homotopic as curves in $\Sigma$. Let $G$ be the 2connected subgraph of $H$ contained in the annulus region of $\Sigma$ between $C_{0}$ and $C_{1}$, including $C_{0}$ and $C_{1}$. Note that $G$ can be viewed as a plane graph with $C_{0}$ and $C_{1}$ as its facial cycles and $C_{1}$ bouding its infinite face. Hence, we call $G$ a cylinder with outer cycle $C_{1}$ and inner cycle $C_{0}$. The cylinder-width of $G$ is the largest integer $q$ such that $G$ contains $q$ pairwise disjoint cycles $R_{0}, \ldots, R_{q-1}$, all homotopic to $C_{0}$ and $C_{1}$ in $\Sigma$.

Suppose that $H$ is embedded in the orientable surface $\Sigma$ of genus $g$, and that $G$ is an induced subgraph of $H$ which is a cylinder with inner cycle $C_{0}$ and outer cycle $C_{1}$. Cutting along both $C_{0}$ and $C_{1}$ produces graphs $H^{\prime}$ and $G$ on some set of surfaces. Here we say that the graph $H^{\prime}$ is produced from $H$ by cutting and deleting the cylinder $G$. Note that if $C_{0}$ and $C_{1}$ are homotopically non-trivial, then $H^{\prime}$ is embedded in a surface $\Sigma^{\prime}$ of genus at most $g-1$. We use 
$S_{g}$ to identify the orientable surface of genus $g$, which has Euler genus $2 g$.

The following result is proven in [8] for triangulations, and is given in [2] as a simple extension to all graphs.

Lemma 1 For any natural numbers $g, r$ there exists a natural number $f(g, r)$ such that any 2-connected graph $H$ in $S_{g}$ having face-width at least $f(g, r)$ contains $g$ pairwise disjoint cylinders $Q_{1}, \ldots, Q_{g}$ of cylinder-width at least $r$ whose cutting and deletion results in a connected plane graph.

Let $C_{i}$ be the outer cycle of $Q_{i}$ and let $D_{i}$ be the inner cycle of $Q_{i}$. Let $Q_{i}^{\prime} \subset Q_{i}$ be a cylinder of $H$ with outer cycle $C_{i}^{\prime}$ and inner cycle $D_{i}^{\prime}$ such that $C_{i}^{\prime}$ and $D_{i}^{\prime}$ are homotopic to $C_{i}$ and $D_{i}$ (as curves in $S_{g}$ ). We observe that, after cutting and deletion of $Q_{1}^{\prime}, \ldots, Q_{g}^{\prime}$ from $H$, the resulting graph is also a connected plane graph. To see this, let $P_{i}$ denote the cylinder of $G$ with outer cycle $C_{i}$ and inner cycle $C_{i}^{\prime}$, and let $R_{i}$ denote the cylinder of $G$ with outer cycle $D_{i}^{\prime}$ and inner cycle $D_{i}$. Assume that $C_{i}^{\prime}$ and $D_{i}^{\prime}$ are choosen such that $P_{i} \cap R_{i}=\emptyset$. Let $G$ be the resulting graph after cutting and deletion of $Q_{1}, \cdots, Q_{g}$ from $H$, and let $G^{\prime}$ be the resulting graph after cutting and deletion of $Q_{1}^{\prime}, \ldots, Q_{g}^{\prime}$ from $H$. Clearly, $G^{\prime}=G \cup\left(\bigcup_{i=1}^{g}\left(P_{i} \cup R_{i}\right)\right)$. Hence, $G^{\prime}$ is also a connected plane graph.

Note that $f(g, r)$ is $O\left(r 4^{g}\right)$ and $f(g, r) \geq 4^{g}$. Using Lemma 1, Böhme, Mohar, and Thomassen [2] proved that there is a function $c(g)$ such that every 3-connected graph $G$ with $n$ vertices embeddable in $S_{g}$ contains a cycle of length at least $c(g) n^{0.4}$. With the result of Chen and $\mathrm{Yu}[3]$, the exponent 0.4 can be improved to $\log _{3} 2 \approx 0.63$. No face-width requirement is placed on the graph $G$, but $c(g)$ depends on the genus of the surface. In this paper we show that by imposing a lower bound on the face-width of $G, c(g)$ can be replaced by a constant which is not dependent on $g$. To do this, we prove a stronger result for weighted graphs.

We will work with graphs which have non-negative vertex weighting. Where 
there is no danger of confusion, we use $v \in G$ to mean $v \in V(G)$. Let $\mathbb{R}^{+}$denote the set of non-negative real numbers. Let $G$ be a graph, and $w: V(G) \rightarrow \mathbb{R}^{+}$. If $H$ is a subgraph of $G$, we define $w(H)=\sum_{v \in H} w(v)$. Define $w(\varnothing)=0$.

We now have the definitions required to precisely state the main result of this paper.

Theorem 1 Let $G$ be a 3-connected graph embedded in $S_{g}$ and having facewidth $\geq f(g, 6)$, and let $w: V(G) \rightarrow \mathbb{R}^{+}$. Then $G$ contains a cycle $R$ such that $\sum_{v \in R} w(v)^{\log _{3} 2} \geq \frac{1}{2}\left[\frac{1}{4} w(G)\right]^{\log _{3} 2}$.

\section{Circuit Graphs - Definitions and Background}

Let us begin with some notation.

Let $S$ be a subgraph of a graph $G$. Denote by $G-S$ the subgraph of $G$ induced by $V(G) \backslash V(S)$. Let $\mathrm{H}$ be a component of $G-S$, and let $B$ be the subgraph induced by $E(H) \cup\{x y \in E(G): x \in V(S), y \in V(H)\}$. Then $B$ is called an $S$-bridge of $G$. We also call a subgraph $B$ an S-bridge of $G$ if $B$ is induced by a single edge $x y \in E(G)-E(S)$ where $x, y \in V(S)$. In both cases, the vertices in $V(B) \cap V(S)$ are called the attachments of $B$.

Let $x, y \in V(G)$ for a graph $G$, and let $P$ be a path in $G$ with $x, y \in V(P)$. By $x P y$ we mean the subpath of $P$ between $x$ and $y$, inclusive. Where $C$ is a cycle in a plane graph $G$ and $x, y \in V(C)$, we use $x C y$ to indicate the subpath of $C$ from $x$ to $y$, inclusive, which follows the clockwise orientation of $C$. Where $x$ and $y$ are the endpoints of a path $R$ in $G$, we call $R$ an $x-y$ path. Let $Y \subset V(G), x \notin Y$. If $R$ is an $x-y$ path with $R \cap Y=\{y\}$, we say that $R$ is an $x-Y$ path. A set of $x-Y$ paths is called an $x-Y$ fan if each pair of the paths have only $x$ in common. If the number of paths in an $x-Y$ fan is $n$, we call it an $x-Y n$-fan. Given a 2-connected plane graph $G$, we call the cycle boundary of the infinite face of $G$ the outer cycle of $G$. 
A circuit graph is a pair $(G, C)$, where $G$ is a 2-connected plane graph and $C$ is a facial cycle of $G$, such that, for every 2-cut $S$ of $G$, every component of $G-S$ contains a vertex of $C$. Given a circuit graph $(G, C)$ and distinct vertices $x, y \in V(C)$, we say that $(G, x C y)$ is a strong circuit graph if, for every 2-cut $S$ of $G, S \cap(y C x-\{x, y\}) \neq \varnothing$.

An annulus graph is a triple $\left(G, C_{1}, C_{2}\right)$, where $G$ is a 2 -connected plane graph, and $C_{1}$ and $C_{2}$ are facial cycles of $G$, such that, for any 2-cut $S$ of $G$, every component of $G-S$ contains a vertex of $C_{1} \cup C_{2}$.

Where $G$ is a connected graph which is not 2-connected, we describe the structure of $G$ using Tutte's block graph construction [10]. A block of $G$ is a maximal 2-connected subgraph. For this purpose, we define any subgraph induced by a single edge to be 2 -connected. We construct a graph $B l k(G)$ such that $V(B l k(G))=\left\{\right.$ cutvertices $u_{i}$ of $\left.G\right\} \cup\left\{\right.$ blocks $B_{j}$ of $\left.G\right\}$, and $u_{i}$ is adjacent to $B_{j}$ in $B l k(G)$ if $u_{i} \in B_{j}$ in $G$. We call a block $B$ an extremal block of $G$ if $B$ contains exactly one cutvertex. If the graph $B l k(G)$ is a simple path, we say that $G$ is a chain of blocks. Note that for a connected graph $G$ this is equivalent to the condition that each block of $G$ contains at most two cutvertices, and each cutvertex of $G$ is contained in at most two blocks. Suppose that $G$ is a chain of blocks, and that each block of $G$ is induced by a single edge or is a circuit graph $(B, C)$ with all cutvertices of $G$ in $B$ lying on the cycle $C$. Then $G$ is called a chain of circuit graphs.

Let $G$ be a chain of circuit graphs such that, for each block $B$ of $G$, one of the following holds:

(i) $B=G$; either $B$ is induced by an edge $x y$, or there is a facial cycle $C$ of $B$ and there are distinct $x, y \in V(C)$ such that $(B, x C y)$ is a strong circuit graph.

(ii) $B \neq G$ is an extremal block containing cutvertex $y$ of $G$; either $B$ is induced by an edge $x y$, or there is a facial cycle $C$ of $G$ containing $y$ and 
there is some $x \in V(C)-\{y\}$ such that one of $(B, x C y)$ or $(B, y C x)$ is a strong circuit graph.

(iii) $B \neq G$ is a non-extremal block containing distinct cutvertices $x, y$ of $G$; either $V(B)=\{x, y\}$ or there is some facial cycle $C$ in $B$ such that $\{x, y\} \in V(C)$ and one of $(B, x C y)$ or $(B, y C x)$ is a strong circuit graph.

We call $G$ a chain of strong circuit graphs.

We will repeatedly make use of the following fact.

Lemma 2 Let $n_{i} \in \mathbb{R}^{+}, i=1, \ldots, m$, and let $0<r \leq 1$. Then $\sum_{i=1}^{m} n_{i}^{r} \geq$ $\left(\sum_{i=1}^{m} n_{i}\right)^{r}$.

Lemma 2 is obvious if $r=1$. So assume $0<r<1$. Define $f(x)=$ $x^{r}+(1-x)^{r}$. One may show that, with $x \in[0,1], f(x)$ has a unique critical point at $x=1 / 2$. Since $f(1 / 2)>1, f(x) \geq 1$ for all $x \in[0,1]$. Now let $x=\frac{n_{1}}{n_{1}+n_{2}}$ to find that $n_{1}^{r}+n_{2}^{r} \geq\left(n_{1}+n_{2}\right)^{r}$, and apply induction on $m$.

Lemma 3 Let $(G, x C y)$ be a strong circuit graph, and let $w: V(G) \rightarrow \mathbb{R}^{+}$. Then $G$ contains an $x-y$ path $P$ such that $\sum_{v \in P-y} w(v)^{\log _{3} 2} \geq w(G-y)^{\log _{3} 2}$.

Lemma 3 is given in [3]. The vertex $y$ is excluded in the above inequality for technical reasons, (counting becomes straightforward when piecing together paths), which will be adopted in this paper. As a warm-up, we prove a similar result for the larger class of circuit graphs.

Lemma 4 Let $(G, C)$ be a circuit graph, let $x, y \in V(C)$ be distinct, and let $w$ : $V(G) \rightarrow \mathbb{R}^{+}$. Then $G$ contains an $x-y$ path $P$ such that $\sum_{v \in P-y} w(v)^{\log _{3} 2} \geq$ $\left[\frac{1}{2} w(G-y)\right]^{\log _{3} 2}$

Proof We assume without loss of generality that $G$ is embedded in the plane with outer cycle $C$. If either of $(G, x C y)$ or $(G, y C x)$ is a strong circuit graph, we simply apply Lemma 3. Let us assume then that neither is a strong circuit 
graph. Hence $G$ has a 2-cut contained in $V(x C y)$ and a 2-cut contained in $V(y C x)$. Let $\left(S_{k}\right)_{k}$ be the collection of 2-cuts of $G$ with $S_{k} \subseteq V(x C y)$, and let $\left(T_{l}\right)_{l}$ be the collection of 2-cuts of $G$ with $T_{l} \subseteq V(y C x)$. Let $B_{k}$ be the union of all $S_{k}$-bridges of $G$ which do not contain $y C x$. Define $H=\left(\bigcup_{k} B_{k}\right)-\{x, y\}$. Similarly define $A_{l}$ as the union of $T_{l}$-bridges of $G$ which do not contain $x C y$, and $L=\left(\bigcup_{l} A_{l}\right)-\{x, y\}$. Since $(G, C)$ is a circuit graph, every componenet of $G-S_{k}$ (respectively $G-T_{l}$ ) contains a vertex of $C$. Hence $H \cap L=\varnothing$.

By symmetry, assume that $w(H) \leq w(L)$. A 2-cut $S_{k} \subseteq V(x C y)$ is called maximal if there is no other 2-cut $S_{j} \subseteq V(x C y)$ for which $B_{k} \subset B_{j}, B_{k} \neq B_{j}$. For each maximal 2-cut $S_{k}$ of $G$, remove the subgraph $B_{k}-S_{k}$ from $G$ and replace it with a single edge $e_{k}$ between the vertices of $S_{k}$ so that the resulting graph is a plane graph. Call the resulting graph $G^{\prime}$, and its outer cycle $C^{\prime}$.

Now $\left(G^{\prime}, x C^{\prime} y\right)$ is a strong circuit graph. To see this, note that any 2-cut $S^{\prime}$ in $G^{\prime}$ would also be a 2-cut in the original graph $G$. Hence $S^{\prime} \subset C^{\prime}$. Note also that since we replaced the bridges of all maximal 2-cuts contained in $V(x C y)$, $S^{\prime} \nsubseteq x C^{\prime} y$. Hence $S^{\prime} \cap\left(y C^{\prime} x-\{x, y\}\right) \neq \varnothing$, and so, $\left(G^{\prime}, x C^{\prime} y\right)$ is a strong circuit graph.

Since $w(H) \leq w(L)$ and $H \cap L=\varnothing$, we have $w\left(G^{\prime}-y\right) \geq \frac{1}{2} w(G-y)$. Applying Lemma 3 to $\left(G^{\prime}, x C^{\prime} y\right)$, we find an $x-y$ path $P^{\prime}$ in $G^{\prime}$ such that

$$
\sum_{v \in P^{\prime}-y} w(v)^{\log _{3} 2} \geq\left[w\left(G^{\prime}-y\right)\right]^{\log _{3} 2} \geq\left[\frac{1}{2} w(G-y)\right]^{\log _{3} 2} .
$$

We then expand $P^{\prime}$ to an $x-y$ path $P$ in $G$ as follows: if $e_{k} \in E\left(P^{\prime}\right)$ and $S_{k}=\{u, v\}$, simply replace $e_{k}$ with any $u-v$ path in $B_{k}$. Now

$$
\sum_{v \in P-y} w(v)^{\log _{3} 2} \geq\left[\frac{1}{2} w(G-y)\right]^{\log _{3} 2} .
$$

Let $G$ be a chain of circuit graphs, and let $B$ be a block of $G$, where $(B, C)$ is a circuit graph or $B$ is induced by a single edge. Call $x \in V(B)$ an outer vertex of $B$ if $x$ is not a cutvertex of $G$, and either $x \in V(C)$ or $x$ has degree 1 in $B$. 
Lemma 5 Let $G$ be a chain of circuit graphs, and let $w: V(G) \rightarrow \mathbb{R}^{+}$. If $G$ has only one block, let $x, y$ be distinct outer vertices of $G$. If $G$ has at least two blocks, let $x \in V(G)$ be an outer vertex of one extremal block of $G$, and let $y \in V(G)$ be an outer vertex of the other extremal block of $G$. Then $G$ contains an $x-y$ path $P$ such that $\sum_{v \in P-y} w(v)^{\log _{3} 2} \geq\left[\frac{1}{2} w(G-y)\right]^{\log _{3} 2}$

Proof Let the blocks of $G$ be labeled by $B_{1}, \ldots, B_{k}$, let the cutvertices be labeled $x_{1}, \ldots, x_{k-1}$, and let $x_{0}=x$ and $x_{k}=y$, so that $x_{i-1}, x_{i} \in B_{i}$ for $i=1, \ldots, k$. To construct the desired long path in $G$, we apply Lemma 4 to each of $B_{i}$, finding an $x_{i-1}-x_{i}$ path $P_{i}$ such that $\sum_{v \in P_{i}-x_{i}} w(v)^{\log _{3} 2} \geq$ $\left[\frac{1}{2} w\left(B_{i}-x_{i}\right)\right]^{\log _{3} 2}$. Let $P=\bigcup_{i=1}^{k} P_{i}$. By applying Lemma 2 , we have

$$
\begin{aligned}
\sum_{v \in P-y} w(v)^{\log _{3} 2}=\sum_{i} \sum_{v \in P_{i}-x_{i}} w(v)^{\log _{3} 2} & \geq \sum_{i}\left[\frac{1}{2} w\left(B_{i}-x_{i}\right)\right]^{\log _{3} 2} \\
& \geq\left[\frac{1}{2} w(G-y)\right]^{\log _{3} 2} .
\end{aligned}
$$

Let us make a few simple observations about the structure of circuit and annulus graphs. These are stated in a fairly weak form, but they will be helpful to simplify the extension of our results to annulus graphs. Where $G$ is a plane graph and $C$ is any cycle in $G$, we define $\operatorname{int}(C)$ to be the subgraph of $G$ contained in the closed disc in the plane which is bounded by $C$.

Lemma 6 Let $G$ be a 2-connected plane graph with outer cycle $C$ and another facial cycle $D$. Let $F$ be any cycle in $G$.

(i) If $(G, C)$ is a circuit graph, then $(\operatorname{int}(F), F)$ is a circuit graph.

(ii) If $(G, C, D)$ is an annulus graph and $D \subseteq \operatorname{int}(F)$, then $(\operatorname{int}(F), F, D)$ is an annulus graph.

(iii) If $(G, C, D)$ is an annulus graph and $D \nsubseteq \operatorname{int}(F)$, then $(\operatorname{int}(F), F)$ is a circuit graph. 
Proof If $\operatorname{int}(F)$ is 3-connected then it is clearly a circuit graph, so we assume that $\operatorname{int}(F)$ is not 3-connected. Let $S$ be an arbitrary 2-cut in $\operatorname{int}(F)$, and let $T$ be any component of $\operatorname{int}(F)-S$.

(i) Assume $(G, C)$ is a circuit graph. Suppose that $T \cap F=\varnothing$, and hence $T \cap C=\varnothing$. Since $G$ is planar, we see that there can be no edges from $T$ to $G-\operatorname{int}(F)$. So $S$ is a 2 -cut in $G$, and $T$ is a component of $G-S$ with $T \cap C=\varnothing$. But this is a contradiction to the hypothesis that $(G, C)$ is a circuit graph. Hence $T \cap F \neq \varnothing$. Since $S$ and $T$ are arbitrary, $(\operatorname{int}(F), F)$ is a circuit graph.

(ii) Suppose $(G, C, D)$ is an annulus graph and $D \subseteq \operatorname{int}(F)$. If $T \cap(F \cup D)=$ $\varnothing$, then $S$ is a 2-cut of $G$ and $T$ is a component of $G-S$, and $T \cap(C \cup D)=$ $\varnothing$, contradicting the assumption that $(G, C, D)$ is an annulus graph. Hence $T \cap(F \cup D) \neq \varnothing$. Since $S$ and $T$ are arbitrary, $(\operatorname{int}(F), F, D)$ is an annulus graph.

(iii) Now suppose $(G, C, D)$ is an annulus graph and $D \nsubseteq \operatorname{int}(F)$. If $T \cap F=$ $\varnothing$ then $S$ is a 2-cut of $G$ and $T$ is a component of $G-S$, and $T \cap(C \cup D)=\varnothing$, contradicting the assumption that $(G, C, D)$ is an annulus graph. Since $S$ and $T$ are arbitrarily chosen, $(\operatorname{int}(F), F)$ must be a circuit graph.

\section{Long Paths in Annulus Graphs}

Lemma 7 Let $(A, C, D)$ be an annulus graph. Let $w: V(A) \rightarrow \mathbb{R}^{+}$, and let $x, y \in V(C \cup D)$ be distinct. Then there is an $x-y$ path $P$ in $A$ such that

$$
\sum_{v \in P-y} w(v)^{\log _{3} 2} \geq\left[\frac{1}{4} w(A-y)\right]^{\log _{3} 2} .
$$

Proof Throughout the proof, we assume without loss of generality that $A$ is embedded in the plane with outer cycle $C$. 


\section{Case $1|C \cap D| \geq 3$.}

We will define two subgraphs of $A$, each of which is a circuit graph or chain of circuit graphs. We begin by defining $U=V(C \cap D)$. Label the vertices of $U$ by $u_{1}, \ldots, u_{m}$ in clockwise order along $C$, and let $u_{m+1}=u_{1}$. Let $M_{i}$ denote the union of $\left\{u_{i}, u_{i+1}\right\}$-bridges of $A$ not containing $u_{i+1} C u_{i} \cup u_{i+1} D u_{i}$.

We claim that there are $M_{k}$ and $M_{l}$ such that either (1) $x \in M_{k}-u_{k+1}$, $y \in M_{l}-u_{l}$, and $u_{k} \neq u_{l+1}$, or $(2) x \in M_{k}-u_{k}, y \in M_{l}-u_{l+1}$, and $u_{l} \neq u_{k+1}$. Assume without loss of generality that $x \in M_{k}-u_{k+1}$ and $y \in M_{l}-u_{l}$. If $u_{k} \neq u_{l+1}$, then (1) holds. So let $u_{k}=u_{l+1}$. Then $u_{k+1} \neq u_{l}$ because $m \geq 3$. If $x \neq u_{k}$ and $y \neq u_{k}$, then we have $x \in M_{k}-u_{k}, y \in M_{l}-u_{l+1}$, and $u_{l} \neq u_{k+1}$, and so, (2) holds. So either $x=u_{k}$ or $y=u_{k}$. If $x=u_{k}$, then $y \neq u_{k}$, and so, $x \in M_{l}-u_{l}, y \in M_{l}-u_{l+1}$, and $u_{l+1} \neq u_{l}$, that is, (2) holds with $M_{k}=M_{l}$. So $y=u_{k}$ and $x \neq u_{k}$. Then $x \in M_{k}-u_{k}, y \in M_{k}-u_{k+1}$, and $u_{k} \neq u_{k+1}$, and so, (2) holds with $M_{l}=M_{k}$.

By symmetry, assume (1) holds (see Figure 1). Let $B_{1}=\cup_{i=k}^{l} M_{i}$, and let $B_{2}=A-\left(B_{1}-\left\{u_{k}, u_{l+1}\right\}\right)$. Note that each $M_{i}$ is either a subgraph of $A$ induced by a single edge or (by (iii) of Lemma 6) a circuit graph with its outer cycle as the special cycle. Hence, $B_{1}$ and $B_{2}$ are chains of circuit graphs. Note that when $k<l, x$ and $y$ lie in distinct extremal blocks of $B_{1}$. Also note that either $w\left(B_{1}-y\right) \geq \frac{1}{2} w(A-y)$ or $w\left(B_{2}-y\right) \geq \frac{1}{2} w(A-y)$; otherwise, we have the contradiction that $w\left(B_{1}-y\right)+w\left(B_{2}-y\right)<w(A-y)$.

(1A) Suppose $w\left(B_{1}-y\right) \geq \frac{1}{2} w(A-y)$. We apply Lemma 5 to find an $x-y$ path $P$ in $B_{1}$ with

$$
\begin{aligned}
\sum_{v \in P-y} w(v)^{\log _{3} 2} & \geq\left[\frac{1}{2} w\left(B_{1}-y\right)\right]^{\log _{3} 2} \\
& \geq\left[\frac{1}{4} w(A-y)\right]^{\log _{3} 2}
\end{aligned}
$$




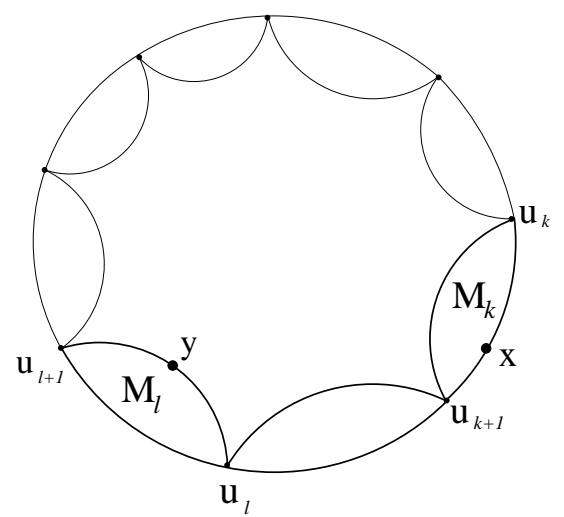

Figure 1: Case 1, $|C \cap D| \geq 3$

(1B) Now suppose $w\left(B_{1}-y\right)<\frac{1}{2} w(A-y)$, so $w\left(B_{2}-y\right) \geq \frac{1}{2} w(A-y)$. By Lemma 5 there is an $u_{k}-u_{l+1}$ path $P_{2}$ in $B_{2}$ with $\sum_{v \in P_{2}-u_{l+1}} w(v)^{\log _{3} 2} \geq$ $\left[\frac{1}{2} w\left(B_{2}-u_{l+1}\right)\right]^{\log _{3} 2}$. Note also that $B_{1}$ contains two disjoint paths $P_{x}, P_{y}$ from $x, y$ to $u_{k}, u_{l+1}$, respectively, which are internally disjoint from $B_{2}$.

We define an $x-y$ path by $P=P_{x} \cup P_{2} \cup P_{y}$. Then, with an application of Lemma 2, we find

$$
\begin{aligned}
\sum_{v \in P-y} w(v)^{\log _{3} 2} & =\sum_{v \in P_{x}-u_{k}} w(v)^{\log _{3} 2}+\sum_{v \in P_{2}-u_{l+1}} w(v)^{\log _{3} 2}+\sum_{v \in P_{y}-y} w(v)^{\log _{3} 2} \\
& \geq \sum_{v \in P_{2}-u_{l+1}} w(v)^{\log _{3} 2}+w\left(u_{l+1}\right)^{\log _{3} 2} \\
& \geq\left[\frac{1}{2} w\left(B_{2}-u_{l+1}\right)\right]^{\log _{3} 2}+w\left(u_{l+1}\right)^{\log _{3} 2} \\
& \geq\left[\frac{1}{2} w\left(B_{2}\right)\right]^{\log _{3} 2} \\
& \geq\left[\frac{1}{4} w(A-y)\right]^{\log _{3} 2}
\end{aligned}
$$

Case $2|C \cap D| \leq 2$.

First we define subgraphs $B_{1}$ and $B_{2}$. Their definitions depend upon the size of $C \cap D$ and are illustrated in Figure 2. 


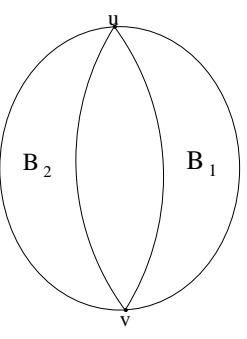

(a) $|C \cap D|=2$

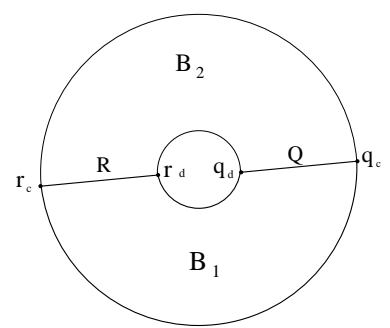

(b) $|C \cap D|=0$

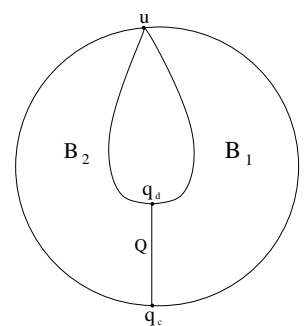

(c) $|C \cap D|=1$

Figure 2: Case 2, $|C \cap D| \leq 2$

When $|C \cap D|=2$ : Let $U=\{u, v\}=C \cap D$. If $u C v=u D v$, then let $B_{1}=u C v$, which is induced by $u v \in E(G)$. Otherwise, let $B_{1}=\operatorname{int}\left(F_{1}\right)$ where $F_{1}=u C v \cup u D v$. If $v C u=v D u$, let $B_{2}=v C u$, which is induced by $u v \in E(G)$. Otherwise, let $B_{2}=\operatorname{int}\left(F_{2}\right)$ where $F_{2}=v C u \cup v D u$.

When $|C \cap D|=0$ : Since $A$ is 2-connected, there must be disjoint paths $Q$ and $R$ from $V(C)$ to $V(D)$. We may assume that each path intersects $C$ and $D$ only at its endpoints. Let $q_{c} \in V(C)$ and $q_{d} \in V(D)$ be the endpoints of $Q$, and $r_{c} \in V(C)$ and $r_{d} \in V(D)$ the endpoints of $R$. Let $U=\left\{q_{c}, q_{d}, r_{c}, r_{d}\right\}$. Define $B_{1}=\operatorname{int}\left(F_{1}\right)$ where $F_{1}=q_{c} C r_{c} \cup Q \cup q_{d} D r_{d} \cup R$. Define $B_{2}=\operatorname{int}\left(F_{2}\right)$ where $F_{2}=r_{c} C q_{c} \cup Q \cup r_{d} D q_{d} \cup R$.

When $|C \cap D|=1$ : Let $\{u\}=C \cap D$. Since $A$ is 2-connected, $A-u$ is connected. We may find a path $Q$ from $V(C)$ to $V(D)$ in $A-u$, such that $Q$ intersects $C$ and $D$ only at its endpoints. Let $\left\{q_{c}\right\}=Q \cap C$ and $\left\{q_{d}\right\}=Q \cap D$. Let $U=\left\{q_{c}, q_{d}, u\right\}$. Define $B_{1}=\operatorname{int}\left(F_{1}\right)$ where $F_{1}=u C q_{c} \cup Q \cup u D q_{d}$. Define $B_{2}=\operatorname{int}\left(F_{2}\right)$ where $F_{2}=q_{c} C u \cup Q \cup q_{d} D u$.

We proceed in the same manner for all three of the above situations.

By the above definitions, $D \nsubseteq B_{1}$ and $D \nsubseteq B_{2}$. From (iii) of Lemma 6 both $\left(B_{1}, F_{1}\right)$ and $\left(B_{2}, F_{2}\right)$ are circuit graphs, except when $B_{1}$ or $B_{2}$ is induced by a 
single edge.

By symmetry, assume that $w\left(B_{1}-y\right) \geq w\left(B_{2}-y\right)$. Then $w\left(B_{1}-y\right) \geq$ $\frac{1}{2} w(A-y)$. In this case, $B_{1}$ cannot be induced by a single edge, and so, $\left(B_{1}, F_{1}\right)$ is a circuit graph.

(2A) First we consider the case $x, y \in B_{1}$. Then by Lemma 4, there is an $x-y$ path $P$ in $B_{1}$ with

$$
\sum_{v \in P-y} w(v)^{\log _{3} 2} \geq\left[\frac{1}{2} w\left(B_{1}-y\right)\right]^{\log _{3} 2} \geq\left[\frac{1}{4} w(A-y)\right]^{\log _{3} 2}
$$

(2B) Now consider the case $x, y \notin B_{1}$, noting that $B_{2}$ may not be induced by a single edge in this case. We recall that $x, y \in V(C \cup D)$. Hence $B_{2}$ contains disjoint paths $P_{x}, P_{y}$ from $x, y$ to vertices $u_{x}, u_{y} \in U$, respectively, with $\left(P_{x} \cup P_{y}\right) \cap U=\left(P_{x} \cup P_{y}\right) \cap B_{1}=\left\{u_{x}, u_{y}\right\}$.

By Lemma 4 there is a $u_{x}-u_{y}$ path $P_{1}$ in $B_{1}$ with

$$
\sum_{v \in P_{1}-u_{y}} w(v)^{\log _{3} 2} \geq\left[\frac{1}{2} w\left(B_{1}-u_{y}\right)\right]^{\log _{3} 2} .
$$

Now we define an $x-y$ path by $P=P_{x} \cup P_{1} \cup P_{y}$. Then, with an application of Lemma 2, we have

$$
\begin{aligned}
\sum_{v \in P-y} w(v)^{\log _{3} 2} & =\sum_{v \in P_{x}-u_{x}} w(v)^{\log _{3} 2}+\sum_{v \in P_{1}-u_{y}} w(v)^{\log _{3} 2}+\sum_{v \in P_{y}-y} w(v)^{\log _{3} 2} \\
& \geq \sum_{v \in P_{1}-u_{y}} w(v)^{\log _{3} 2}+w\left(u_{y}\right)^{\log _{3} 2} \\
& \geq\left[\frac{1}{2} w\left(B_{1}-u_{y}\right)\right]^{\log _{3} 2}+w\left(u_{y}\right)^{\log _{3} 2} \\
& \geq\left[\frac{1}{2} w\left(B_{1}\right)\right]^{\log _{3} 2} \\
& \geq\left[\frac{1}{4} w(A-y)\right]^{\log _{3} 2} .
\end{aligned}
$$


(2C) Finally, suppose $x \notin B_{1}, y \in B_{1}$. The case $x \in B_{1}, y \notin B_{1}$ is symmetric.

Since $x \in V(C \cup D), B_{2}$ contains a path $P_{2}$ from $x$ to some vertex $u_{x} \in U$, such that $P_{2} \cap U=P_{2} \cap B_{1}=\left\{u_{x}\right\}$.

By Lemma 4 , there is a $u_{x}-y$ path $P_{1}$ in $B_{1}$ with $\sum_{v \in P_{1}-y} w(v)^{\log _{3} 2} \geq$ $\left[\frac{1}{2} w\left(B_{1}-y\right)\right]^{\log _{3} 2}$.

Now we define an $x-y$ path by $P=P_{1} \cup P_{2}$. Then

$$
\begin{aligned}
\sum_{v \in P-y} w(v)^{\log _{3} 2} & \geq \sum_{v \in P_{1}-y} w(v)^{\log _{3} 2} \\
& \geq\left[\frac{1}{2} w\left(B_{1}-y\right)\right]^{\log _{3} 2} \\
& \geq\left[\frac{1}{4} w(A-y)\right]^{\log _{3} 2}
\end{aligned}
$$

\section{Proof of Theorem 1}

Given a 3-connected graph $G$ embedded in $S_{g}$ with sufficiently large face-width, we apply Lemma 1 to create a connected plane graph $G^{\prime}$. We do not use the graph $G^{\prime}$ directly. Instead, we use observations about the cutting cylinders used to create $G^{\prime}$ from $G$, to construct from $G$ a 3 -connected plane graph $H$. With the results of [3] we find that $H$ has a heavy cycle. A modification of this cycle is then used to produce a heavy cycle in the original graph $G$.

Where $S, T \subset V(G)$ for a graph $G$, we define $[S, T]=\{x y \in E(G): x \in$ $S, y \in T\}$.

(1) Selecting the Cutting Cylinders Let $G$ be a 3-connected graph embedded in $S_{g}$ with face-width at least $f(g, 6)$. Then by Lemma $1, G$ contains $g$ pairwise disjoint cylinders $Q_{1}, \ldots, Q_{g}$ of cylinder-width at least six whose cutting and deletion results in a connected plane graph $G^{\prime}$. Let $C_{i}^{0}$ and $C_{i}^{5}$ be the cycles in $Q_{i}, i=1, \ldots, g$ along which we cut, with $C_{i}^{0}$ the inner cycle of $Q_{i}$ and $C_{i}^{5}$ its outer cycle. Note that each of these cutting cycles is homotopically 
nontrivial in $S_{g}$ (because otherwise $G^{\prime}$ is not connected). By the observation following Lemma 1 , we may choose $Q_{1}, \ldots, Q_{g}$ such that any cylinder of $G$ contained in $Q_{i}$ with outer cycle and inner cycle homotopic to $C_{i}^{0}$ and $C_{i}^{5}$ have cylinder-width $<6$.

In the following discussion, where $C$ is a cycle in $G$ we will also use $C$ to refer to the corresponding cycle in $G^{\prime}$ or in $Q_{i}$. Let us view each of the deleted cylinders, $Q_{i}$, as a plane graph with $C_{i}^{5}$ as its outer cycle and $C_{i}^{0}$ as a facial cycle. Since $Q_{i}$ has cylinder-width at least six, there exist disjoint cycles $C_{i}^{1}, C_{i}^{2}, C_{i}^{3}, C_{i}^{4}$ in $Q_{i}$, also disjoint from $C_{i}^{0}$ and $C_{i}^{5}$, with $C_{i}^{0} \subseteq \operatorname{int}\left(C_{i}^{1}\right) \subset \ldots \subseteq$ $\operatorname{int}\left(C_{i}^{4}\right) \subseteq \operatorname{int}\left(C_{i}^{5}\right)$. We choose $C_{i}^{1}, C_{i}^{2}, C_{i}^{3}, C_{i}^{4}$ such that the following graphs are minimal: $\operatorname{int}\left(C_{i}^{1}\right), \operatorname{int}\left(C_{i}^{3}\right)-\left(\operatorname{int}\left(C_{i}^{2}\right)-C_{i}^{2}\right)$, and $\operatorname{int}\left(C_{i}^{5}\right)-\left(\operatorname{int}\left(C_{i}^{4}\right)-C_{i}^{4}\right)$.

Claim 1 There are no vertices between $C_{i}^{0}$ and $C_{i}^{1}$, between $C_{i}^{2}$ and $C_{i}^{3}$, and between $C_{i}^{4}$ and $C_{i}^{5}$. More precisely, $V\left(\operatorname{int}\left(C_{i}^{1}\right) \backslash \operatorname{int}\left(C_{i}^{0}\right)\right)=V\left(C_{i}^{1}\right), V\left(\operatorname{int}\left(C_{i}^{3}\right) \backslash\right.$ $\left.\operatorname{int}\left(C_{i}^{2}\right)\right)=V\left(C_{i}^{3}\right)$, and $V\left(\operatorname{int}\left(C_{i}^{5}\right) \backslash \operatorname{int}\left(C_{i}^{4}\right)\right)=V\left(C_{i}^{5}\right)$.

Proof Suppose there is some vertex $v \in \operatorname{int}\left(C_{i}^{1}\right) \backslash \operatorname{int}\left(C_{i}^{0}\right)$ with $v \notin V\left(C_{i}^{1}\right)$. Since $G$ is 3 -connected, there is a $v-V\left(C_{i}^{0} \cup C_{i}^{1}\right) 3$-fan in $\operatorname{int}\left(C_{i}^{1}\right)$. That is, there are three distinct paths from $v$ to the set $V\left(C_{i}^{0} \cup C_{i}^{1}\right)$ which are disjoint except for $x$ and which intersect $V\left(C_{i}^{0} \cup C_{i}^{1}\right)$ only at their endpoints. Two of these paths, say $P$ and $Q$, must end on the same cycle. Let $p, q$ be the endpoints of $P, Q$, respectively, other than $v$.

Case $1 . p, q \in C_{i}^{1}$.

Then either $C_{i}^{0} \subset \operatorname{int}\left(P \cup Q \cup p C_{i}^{1} q\right)$ or $C_{i}^{0} \subset \operatorname{int}\left(P \cup Q \cup q C_{i}^{1} p\right)$. Assume by symmetry that $C_{i}^{0} \subset \operatorname{int}\left(P \cup Q \cup p C_{i}^{1} q\right)$. Since $E\left(q C_{i}^{1} p\right)$ is outside $\operatorname{int}(P \cup Q \cup$ $\left.p C_{i}^{1} q\right), \operatorname{int}\left(C_{i}^{1}\right)$ is properly contained in $\operatorname{int}\left(P \cup Q \cup p C_{i}^{1} q\right)$. Note that $P \cup Q \cup p C_{i}^{1} q$ is disjoint from $C_{i}^{0}, C_{i}^{2}, C_{i}^{3}, C_{i}^{4}, C_{i}^{5}$. Hence, $P \cup Q \cup p C_{i}^{1} q$ contradicts the choice of $C_{i}^{1}$. 
Case $2 \cdot p, q \in C_{i}^{0}$.

Then either $C_{i}^{0} \subset \operatorname{int}\left(P \cup Q \cup p C_{i}^{0} q\right)$ or $C_{i}^{0} \subset \operatorname{int}\left(P \cup Q \cup q C_{i}^{0} p\right)$. By symmetry, assume that $C_{i}^{0} \subset \operatorname{int}\left(P \cup Q \cup p C_{i}^{0} q\right)$, and let $C_{i}^{\prime}=P \cup Q \cup p C_{i}^{0} q$. Let $Q_{i}^{\prime}$ be the cyclinder of $G$ with outer cycle $C_{i}^{5}$ and inner cycle $C_{i}^{\prime}$. Clearly, $C_{i}^{\prime}$ is homotopic to $C_{i}^{0}$ in $S_{g}$, and $Q_{i}^{\prime}$ has cylinder width 6 , contradicting the choice of $Q_{i}$.

Hence, we proved that $V\left(\operatorname{int}\left(C_{i}^{1}\right) \backslash \operatorname{int}\left(C_{i}^{0}\right)\right)=V\left(C_{i}^{1}\right)$. By a similar argument, we can prove $V\left(\operatorname{int}\left(C_{i}^{5}\right) \backslash \operatorname{int}\left(C_{i}^{4}\right)\right)=V\left(C_{i}^{5}\right)$. By an argument similar to Case 1 , we can show that $V\left(\operatorname{int}\left(C_{i}^{3}\right) \backslash \operatorname{int}\left(C_{i}^{2}\right)\right)=V\left(C_{i}^{3}\right)$.

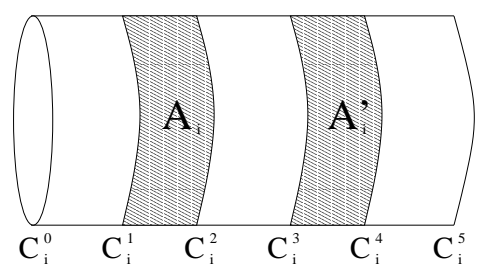

Figure 3: Cutting cylinder $Q_{i}$

Let $A_{i}=\operatorname{int}\left(C_{i}^{2}\right)-C_{i}^{0}$. Then $\left(A_{i}, C_{i}^{2}, C_{i}^{1}\right)$ can be viewed as an annulus graph. (That is, it has a natural planar embedding with outer cycle $C_{i}^{2}$ which is an annulus graph). Let $A_{i}^{\prime}=\operatorname{int}\left(C_{i}^{4}\right)-\operatorname{int}\left(C_{i}^{2}\right)$, with $\left(A_{i}^{\prime}, C_{i}^{4}, C_{i}^{3}\right)$ also seen as an annulus graph. (See Figure 3.)

(2) Constructing the New Graph Recall that $G^{\prime}$ is the connected plane graph obtained from $H$ by cutting and deleting $Q_{1}, \ldots, Q_{g}$.

Claim $2 G^{\prime}$ is 2-connected, and for any 2-cut $S$ of $G^{\prime}$, (1) $S \subset V\left(C_{i}^{0}\right)$ or $S \subset V\left(C_{i}^{5}\right)$ for some $1 \leq i \leq q$, and (2) $G^{\prime}-S$ has exactly two components, each containing a vertex of $C_{i}^{0}$ if $S \subset V\left(C_{i}^{0}\right)$ or $C_{i}^{1}$ if $S \subset V\left(C_{i}^{1}\right)$.

Proof Let $S \subset V\left(G^{\prime}\right)$ be a cutset in $G^{\prime}$ with $|S| \leq 2$. Let $T_{1}, \ldots, T_{k}$ be the components of $G^{\prime}-S$. For each $j \in\{1, \ldots, k\}$, there is a simple closed curve 
$\gamma_{j}$ in the plane such that $\gamma_{j} \cap G^{\prime}=S$, and $T_{j}$ is contained in one open region of the plane bounded by $\gamma_{j}$ while $\bigcup_{i \neq j} T_{i}$ is contained in the other open region bounded by $\gamma_{j}$. Let $\Gamma_{j}$ denote the simple closed curve in $S_{g}$ corresponding to $\gamma_{j}$. Then $\Gamma_{j} \cap G=S$. Since $G$ has face-width greater than three, $\Gamma_{j}$ must be homotopically trivial in $S_{g}$. Since $G$ is 3-connected, we see that $T_{j} \cap D_{j} \neq \varnothing$ for some cycle $D_{j} \in\left\{C_{i}^{0}, C_{i}^{5}: 1 \leq i \leq q\right\}$. But since $D_{j}$ is homotopically non-trivial, no $D_{j}$ is contained in the open region in the plane bounded by $\gamma_{j}$ and containing $T_{j}$. Hence $S \subset D_{j}$ for all $j \in\{1, \ldots, k\}$. This forces $k=2$ and $|S|=2$. This proves the claim.

Now we turn to the construction of the graph $H$. Beginning from $G$, remove all $\left[C_{i}^{2}, C_{i}^{3}\right], i=1, \ldots, g$. Contract each subgraph $A_{i}$ to the vertex $a_{i}$ and contract each $A_{i}^{\prime}$ to the vertex $a_{i}^{\prime}$, deleting multiple edges, and letting $\tilde{w}\left(a_{i}\right)=$ $w\left(A_{i}\right), \tilde{w}\left(a_{i}^{\prime}\right)=w\left(A_{i}^{\prime}\right)$, for $i=1, \ldots, g$. Let $\tilde{w}(x)=w(x)$ for all $x \in V(G-$ $\left.\left(A_{i} \cup A_{i}^{\prime}\right)\right)$. Call the resulting graph $H$. Then $\tilde{w}(H)=w(G)$. Note that $G-\bigcup_{i=1}^{g}\left(A_{i} \cup A_{i}^{\prime}\right)=G^{\prime}$. By Claim 2 and since the face-width of $G$ is at least three, we see that $H$ is a 3 -connected plane graph.

(3) Constructing a Long Cycle Let $C$ be a facial cycle of $H$, and let $e=x y \in E(C)$. Since $H$ is 3-connected, $(H, x C y)$ is a strong circuit graph. Applying Lemma 3 we find that there is some $x-y$ path $P$ in $H$ with

$$
\sum_{v \in P-y} \tilde{w}(v)^{\log _{3} 2} \geq \tilde{w}(H-y)^{\log _{3} 2}
$$

Note that we may choose $P$ so that $V(P) \neq\{x, y\}$. Now let $T$ be the cycle in $H$ obtained from $P$ by adding the edge $e$. By Lemma 2, we find that

$$
\sum_{v \in T} \tilde{w}(v)^{\log _{3} 2} \geq \tilde{w}(H-y)^{\log _{3} 2}+\tilde{w}(y)^{\log _{3} 2} \geq \tilde{w}(H)^{\log _{3} 2} .
$$

However, the cycle $T$ may pass through contracted vertices $a_{i}$ or $a_{i}^{\prime}$.

Note that any cycle $T$ in $H$ which passes through the contracted vertex $a_{i}$ can be easily extended to a cycle in $G$ which passes through the annulus 
subgraph $A_{i}$. To reach $a_{i}$ in $H, T$ must pass through two distinct vertices $u_{i}, v_{i}$ on the cycle surrounding $a_{i}$, which corresponds to the cutting cycle $C_{i}^{0}$ in $G$. We will call vertex $a_{i}$ good in cycle $T$ if there exist edges $u_{i} x_{i}, v_{i} y_{i} \in E(G)$ with $x_{i}, y_{i}$ distinct vertices on the cycle $C_{i}^{1}$. If no such edges exist, we call $a_{i}$ bad in $T$. Similar definitions are used for $a_{i}^{\prime}$, with respect to $C_{i}^{4}$ and $C_{i}^{5}$. (Note that if $a_{i} \notin V(T)$, then $a_{i}$ is considered neither good nor bad in $T$.)

The notation of the following discussion will be simplified by a re-indexing of the vertices $a_{i}^{\prime}$. We let $a_{i+g}=a_{i}^{\prime}$ for $i=1, \ldots, g$, so that $\left\{a_{i}\right\}_{i=1}^{2 g}=\left\{a_{i}\right\}_{i=1}^{g} \cup$ $\left\{a_{i}^{\prime}\right\}_{i=1}^{g}$. Similarly let $A_{i+g}=A_{i}^{\prime}$ for $i=1, \ldots, g$. Define $W=T-\left\{a_{i}\right\}_{i=1}^{2 g}$. Let $I=\left\{i \in \mathbb{Z}: a_{i}\right.$ is good in $\left.T\right\}, J=\left\{j \in \mathbb{Z}: a_{j}\right.$ is bad in $\left.T\right\}$.

We construct a cycle $R$ in $G$ based on the cycle $T$ in $H$ as follows. For each $i \in I$, we may apply Lemma 7 to find an $x_{i}-y_{i}$ path $R_{i}$ in $A_{i}$ with $\sum_{v \in R_{i}-y_{i}} w(v)^{\log _{3} 2} \geq\left[\frac{1}{4} w\left(A_{i}-y_{i}\right)\right]^{\log _{3} 2}$. For each $j \in J$, let $x_{j} \in A_{j}$ with $u_{j} x_{j}, v_{j} x_{j} \in E(G)$. Define the cycle $R$ in $G$ as $W \cup\left(\bigcup_{i \in I} u_{i} x_{i} R_{i} y_{i} v_{i}\right) \cup\left(\bigcup_{j \in J} u_{j} x_{j} v_{j}\right)$.

Note that from our choice of the original cycle $T$ in $H$, we have

$$
\begin{aligned}
\sum_{v \in W} \tilde{w}(v)^{\log _{3} 2}+ & \sum_{i \in I} \tilde{w}\left(a_{i}\right)^{\log _{3} 2}+\sum_{j \in J} \tilde{w}\left(a_{j}\right)^{\log _{3} 2} \\
& =\sum_{v \in T} \tilde{w}(v)^{\log _{3} 2} \\
& \geq \tilde{w}(H)^{\log _{3} 2} \\
& =w(G)^{\log _{3} 2} .
\end{aligned}
$$

So considering the weight of the new cycle $R$, with an application of Lemma 2, we have 


$$
\begin{aligned}
\sum_{v \in R} w(v)^{\log _{3} 2} & =\sum_{v \in W} w(v)^{\log _{3} 2}+\sum_{i \in I} \sum_{v \in R_{i}-y_{i}} w(v)^{\log _{3} 2}+\sum_{i \in I} w\left(y_{i}\right)^{\log _{3} 2}+\sum_{j \in J} w\left(x_{j}\right)^{\log _{3} 2} \\
& \geq \sum_{v \in W} w(v)^{\log _{3} 2}+\sum_{i \in I}\left[\frac{1}{4} w\left(A_{i}-y_{i}\right)\right]^{\log _{3} 2}+\sum_{i \in I}\left[\frac{1}{4} w\left(y_{i}\right)\right]^{\log _{3} 2} \\
& \geq\left[\frac{1}{4}\right]^{\log _{3} 2}\left[\sum_{v \in W} \tilde{w}(v)^{\log _{3} 2}+\sum_{i \in I} \tilde{w}\left(a_{i}\right)^{\log _{3} 2}\right] .
\end{aligned}
$$

If we have $\sum_{v \in W} \tilde{w}(v)^{\log _{3} 2}+\sum_{i \in I} \tilde{w}\left(a_{i}\right)^{\log _{3} 2} \geq \frac{1}{2} \sum_{v \in T} \tilde{w}(v)^{\log _{3} 2}$ then we find

$$
\sum_{v \in R} w(v)^{\log _{3} 2} \geq \frac{1}{2}\left[\frac{1}{4} w(G)\right]^{\log _{3} 2} .
$$

We may assume then that

$$
\sum_{v \in W} \tilde{w}(v)^{\log _{3} 2}+\sum_{i \in I} \tilde{w}\left(a_{i}\right)^{\log _{3} 2}<\frac{1}{2} \sum_{v \in T} \tilde{w}(v)^{\log _{3} 2} .
$$

So we must have

$$
\sum_{j \in J} \tilde{w}\left(a_{j}\right)^{\log _{3} 2}>\frac{1}{2} \sum_{v \in T} \tilde{w}(v)^{\log _{3} 2} .
$$

In this case, we will show that $H$ contains a cycle $T^{\prime}$ through all $a_{j}$ with $j \in J$ such that $a_{j}$ is good with respect to $T^{\prime}$. That is, if $u_{j} a_{j} v_{j} \subset T^{\prime}$, then $\exists x_{j}, y_{j} \in V\left(C_{j}^{1}\right)$ (or $V\left(C_{j-g}^{4}\right)$ if $j \geq g+1$ ) such that $x_{j} \neq y_{j}$ and $u_{j} x_{j}, y_{j} v_{j} \in$ $E(G)$.

For each $j \in J$, consider the bipartite graph $G_{j}$ induced by $\left[C_{j}^{0}, C_{j}^{1}\right]$ (or by $\left[C_{j-g}^{4}, C_{j-g}^{5}\right]$ if $\left.j \geq g+1\right)$. Let $V_{j}$ be a vertex cover for $G_{j}$. Then we can find a homotopically non-trivial closed curve $\Gamma_{j}$ lying between $C_{j}^{0}$ and $C_{j}^{1}$ (or $C_{j-g}^{4}$ and $\left.C_{j-g}^{5}\right)$ in $S_{g}$ with $\Gamma_{j} \cap G=V_{j}$. The face-width of $G$ is at least $f(g, 6)$, so $\left|V_{j}\right| \geq f(g, 6)$. Applying Konig's theorem we find that there must be a matching $M_{j}$ in $G_{j}$ with $\left|M_{j}\right| \geq f(g, 6)$. Let $H^{\prime}$ be obtained from $H$ by deleting edges in $\left[C_{j}^{0}, C_{j}^{1}\right]-M_{j}\left(\right.$ or $\left[C_{j-g}^{4}, C_{j-g}^{5}\right]-M_{j}$ if $\left.j \geq g+1\right)$. 
Note that $|J| \leq 2 g$ and $f(g, 6)$ as specified in Lemma 1 is greater than $4 g$. Note also that $H^{\prime}$ has no set $S$ with $|S|<f(g, 6)$ separating two vertices of $\left\{a_{j}: j \in J\right\}$, otherwise the face-width of $G$ is $<f(g, 6)$. Hence $H^{\prime}$ contains a cycle $T^{\prime}$ through all $a_{j}, j \in J$. By the construction of $H^{\prime}, T^{\prime}$ is a cycle of $H$ through all $a_{j}, j \in J$, and the edges of $T^{\prime}$ at $a_{j}, j \in J$, correspond to edges in $M_{j}$. Since $M_{j}$ is a matching in $G$, every $a_{j}$ is good with respect to $T^{\prime}$.

Now, let $u_{j}, v_{j}$ be the neighbors of $a_{j}$ in $T^{\prime}$, and let $x_{j} \neq y_{j} \in V\left(C_{j}^{1}\right)$ (or $V\left(C_{j-g}^{4}\right)$ if $\left.j \geq g+1\right)$ such that $x_{j} u_{j}, y_{j} v_{j} \in E(G)$. For $j \in J$, applying Lemma 7 to $A_{j}$ we find an $x_{j}-y_{j}$ path $R_{j}$ in $A_{j}$ such that

$$
\sum_{v \in R_{j}-y_{j}} w(v)^{\log _{3} 2} \geq\left[\frac{1}{4} w\left(A_{j}-y_{j}\right)\right]^{\log _{3} 2}
$$

Define the cycle $R^{\prime}$ in $G$ as $\left(T^{\prime}-\left\{a_{j}: j \in J\right\}\right) \cup\left(\bigcup_{j \in J} u_{j} x_{j} R_{j} y_{j} v_{j}\right)$.

We then have, again with an application of Lemma 2 ,

$$
\begin{aligned}
\sum_{v \in R^{\prime}} w(v)^{\log _{3} 2} & \geq \sum_{j \in J} \sum_{v \in R_{j}-y_{j}} w(v)^{\log _{3} 2}+\sum_{j \in J} w\left(y_{j}\right)^{\log _{3} 2} \\
& \geq \sum_{j \in J}\left[\frac{1}{4} w\left(A_{j}-y_{j}\right)\right]^{\log _{3} 2}+\sum_{j \in J}\left[\frac{1}{4} w\left(y_{j}\right)\right]^{\log _{3} 2} \\
& \geq \sum_{j \in J}\left[\frac{1}{4} \tilde{w}\left(a_{j}\right)\right]^{\log _{3} 2} \\
& \geq \frac{1}{2}\left[\frac{1}{4} w(G)\right]^{\log _{3} 2}
\end{aligned}
$$




\section{References}

[1] D. Archdeacon and N. Hartsfield and C.H.C. Little, Nonhamiltonian triangulations with large connectivity and representativity, J. Combin. Theory Ser B. 68 (1996) 45-55

[2] T. Bohme and B. Mohar and C. Thomassen, Long cycles in graphs on a fixed surface, University of Ljubljana, Institute of Mathematics, Physics, and Mechanics, Dept. of Mathematics, Preprint Series, 37 no 649 (1999).

[3] G. Chen and X. Yu, Long cycles in 3-connected graphs, submitted.

[4] Z. Gao and X. Yu, Convex programming and circumference of 3-connected graphs of low genus, J. Combin. Theory Ser. B 69 (1997) 39-51.

[5] B. Jackson and N. Wormwald, Longest cycles in 3-connected planar graphs, J. Combin. Theory Ser. B 54 (1992) 291-321.

[6] J. W. Moon and L. Moser, Simple paths on polyhedra, Pacific J. Math. 13 (1963) 629-631.

[7] R. Thomas and X. Yu, 4-connected projective planar graphs are hamiltonian, J. Combin. Theory Ser. B 62 (1994) 114-132.

[8] C. Thomassen, Color-critical graphs on a fixed surface, J. Combin. Theory Ser. B 70 (1997) 67-100.

[9] W.T. Tutte, A theorem on planar graphs, Trans. Amer. Math. Soc. 82 (1956) 99-116.

[10] W.T. Tutte, Graph Theory, Addison-Wesley Publishing Co., 1984.

[11] H. Whitney, A theorem on graphs, Ann. of Math. 32 (1931) 378-390.

[12] X. Yu, Disjoint paths, planarizing cycles, and spanning walks, Trans. Amer. Math. Soc. vol 349 no 4 (1997) 1333-1358. 